\title{
ESQUEMAS DE PENSAMIENTO DE AUTODIÁLOGO POSITIVO Y NEGATIVO Y ESTILOS DE APRENDIZAJE EN ESTUDIANTES UNIVERSITARIOS
}

\author{
Oswaldo Orellana M.,' Lupe Garcia A., Marina Salazar C., Carmela Malaver S., Edgar Herrera F., Elisa Yanac \\ R., Gloria Diaz A., Jorge Rivera M., Martha Zegarra G., Daphne Orellana G. Genaro Araujo S. \\ Universidad Nacional Mayor de San Marcos, Lima, Perú \\ (RECiBIDO el 10/11/2009, ACEPTADo el 11/12/2009)
}

\begin{abstract}
RESUMEN
La investigación es de carácter descriptivo comparativo y tiene como objetivo dar a conocer los resultados de una investigación psicológica sobre los esquemas de pensamiento de autodiálogo, positivo y negativo y estilos de aprendizaje de los estudiantes de San Marcos pertenecientes a cada una de las cinco áreas académicas que organizan la formación profesional: Ciencias de la Salud, Ciencias Básicas, Ingenierías, Ciencias Empresariales y Humanidades.

Para los efectos del estudio se ha empleado dos instrumentos: (1) Inventario de Autodiálogo (Calvete, Estevés, Landin), que comprende dos subescalas: positivo y negativo, ambas con las especificaciones técnicas para poder inferir resultados válidos y confiables; así tenemos los estadísticos originales, los coeficientes alpha del 0.89 para las escalas de autodiálogo negativo y $0.86,0.79$ y 0.87 para las subescalas de pensamientos relacionados con la ansiedad, depresión e ira, respectivamente. De otro lado, los coeficientes alpha fueron 0.82 para la Escala de autodiálogo positivo y $0.72,0.76$ y 0.55 para las subescalas de minimización, afecto positivo y autoinstrucciones de afrontamiento respectivamente; (2) Cuestionario HoneyAlonso de Estilos de Aprendizaje (CHAEA) que investiga cuatro estilos de aprendizaje: activo, reflexivo, teórico y pragmático, con los consiguientes criterios de validez, 0.84 (teórico), 0.82 (reflexivo), 0.78 (pragmático) y 0.74 (activo).
\end{abstract}

La muestra estuvo constituida por 292 estudiantes, distribuidos de la siguiente manera: área de Ciencias de la Salud: 96, área de Ciencias Básicas: 44; área de Ingenierías: 67, área de Ciencias Empresariales: 52 y Área de Letras y Humanidades: 33. Por el análisis de consistencia, correlación y mapa de correspondencias, los instrumentos empleados posibilita demostrar la pertinencia para una aproximación al conocimiento de cómo se expresa el autodiálogo positivo y negativo y los estilos de aprendizaje en estudiantes universitarios, particularmente en la población investigada.

Sin embargo, es preciso indicar que los procesos cognitivos del autodiálogo y los estilos de aprendizaje son procesos independientes, es decir, que no se encuentra una asociación directa entre ambos, sino que cada uno tiene sus manifestaciones específicas en las personas. La edad y el género tampoco se encuentra asociada a un tipo específico de autodiálogo o estilo de aprendizaje. Existen manifestaciones diferentes en las asociaciones de los autodiálogos, positivo y negativo y los estilos de aprendizaje, en las cinco áreas académicas de la población investigada. En el área de las Ciencias Básicas existen asociaciones inversas entre el estilo de aprendizaje pragmático y el estilo de aprendizaje reflexivo, predominando

1 Docente principal de la Facultad de Psicología UNMSM. E-mail: orellanaoswaldo@gmail.com 
el autodiálogo positivo: minimización; en el área Económico-Empresarial, existe una asociación inversa en el autodiálogo negativo, referida específicamente a la depresión y a la ansiedad; paradójicamente en un segundo nivel, la ansiedad y la depresión pueden regularse recíprocamente, destacando diferentemente una asociación entre el autodiálogo negativo, ansiedad, con el estilo de aprendizaje teórico; en el área de Humanidades existe una relación directa del estilo de aprendizaje reflexivo, aunque hay una tendencia a tener una relación inversa al estilo de aprendizaje pragmático; en el área de Ingenierías existe una relación inversa del estilo de aprendizaje activo con el estilo de aprendizaje teórico; finalmente en el área de Ciencias de la Salud existe una relación entre el estilo de aprendizaje activo y el autodiálogo de afecto positivo.

Palabras clave: Autodiálogo positivo, autodiálogo negativo, estilos de aprendizaje, áreas académicas universitarias.

\section{ABSTRACT \\ KNOWLEDGE'S SCHEMES OF POSITIVE AND NEGATIVE SELF-TALK LEARNING STYLES IN UNIVERSITY STUDENTS}

The research is descriptive and comparative aims to disseminate the results of psychological research on mindsets of self-talk, positive and negative, and learning styles of students in San Marcos belonging to each of the five academic field organizing vocational training: Health Sciences, Basic Sciences, Engineering, Business and Humanities.

For purposes of the study has used two instruments: (1) self-talk Inventory (Calvete, Esteve, Landin), comprising two subscales: positive and negative, both with the technical specifications in order to draw conclusions valid and reliable, so we the original statistics, alpha coefficients of 0.89 for negative self-talk scales and $0.86,0.79$ and 0.87 for the subscales of thoughts related to anxiety, depression and anger, respectively. On the other hand, the alpha coefficients were 0.82 for positive self-Scale and $0.72,0.76$ and 0.55 for the subscales of minimization, positive affect and coping self-talk, respectively, (2) HoneyAlonso Questionnaire Learning Styles (CHAEA) investigating four learning styles: active, reflective, theoretical and pragmatic, with consequent acceptance criteria, 0.84 (theoretical), 0.82 (reflective), 0.78 (pragmatic) and 0.74 (active).

The sample consisted of 292 students, distributed as follows: area Health Sciences: 96, area Basic Sciences: 44; area of Engineering: 67 Business area 52 and area Arts and Humanities: 33. For the consistency analysis, correlation and correlation map, the instruments used to demonstrate the relevance enables an approach to the knowledge of how to express the positive and negative self-talk and learning styles in college students, particularly in the research population. However it is noted that the cognitive processes of self-talk and learning styles are independent processes, ie not found a direct association between the two, but each has its specific manifestations in people. The age and gender is associated ma not a specific type of learning style self-talk or different manifestations exist in the associations of self-talk, positive and negative, and learning styles in five academic areas the population. In the field of basic science are inverse associations between learning style pragmatic and reflective learning style, dominating the positive self-talk: minimization. In the economic field business, there is an inverse association in the negative self-talk, specifically referred to depression and anxiety, paradoxically at a second level, anxiety and depression can regulate each other, highlighting different association between negative self-talk, anxiety, with learning style theory. In the humanities there is a direct reflective learning style, although there is a tendency to have an inverse relationship to pragmatic learning style. In the field of engineering an inverse relationship of active learning style with learning style theory. In 
the field of health sciences there is a relationship between the style of active learning and self-talk of positive affect.

Keywords: positive self-talk, negative self-talk, learning styles, university academic areas.

\section{INTRODUCCIÓN}

Los trabajos más importantes acerca del estudio del pensamiento y los estilos de aprendizaje provienen de los distintos formatos de la psicología cognitiva, cuyas expresiones se realizan en las áreas de psicología educacional y psicología clínica.

En la Psicología encontramos una variedad de estudios sobre los pensamientos elaborados hacía sí mismo, así tenemos las autoafirmaciones (Uhlemann y Plater, 1990), que utilizan cinco categorías cognitivas: (1) pensamientos sobre uno mismo, (2) pensamientos sobre la interacción, (3) pensamientos sobre los otros individuos implicados, (4) pensamientos irrelevantes y (5) autodiálogo de clasificación.

De otro lado, la mente ha sido estudiada desde distintas estructuras teóricas: las propuestas cognitivas psicogenéticas (Piaget), histórico cultural (Vigotsky, Luria y Leontiev), el funcionamiento cognitivo de la depresión (Beck), la experiencia de la infancia en el trastorno cognitivo (Bowlby).

Por su parte, Young $(1994,1995)$ establece que los esquemas cognitivos están organizados en cinco grandes dominios o categorías: (1) Dominio de desconexión o rechazo, (2) Dominio de autonomía deteriorada, (3) Dominio de límite deteriorado, (4) Dominio de orientación a los demás, y (5) Dominio de vigilancia excesiva e inhibición. Revisemos cada uno de ellos. La desconexión y rechazo establece las referencias sobre las dificultades de cubrir las expectativas de las necesidades de seguridad, aceptación y respeto; los esquemas que se forman en este dominio son el abandono/inestabilidad, privación emocional, abuso e imperfección/culpa; esquemas que de acuerdo a Mc Ginn y Young (1998) son resultado de experiencias tempranas de separación y rechazo o de ambientes familiares fríos, impredecibles o abusadores. El dominio a la autonomía deteriorada hace referencia a la visión negativa de sí mismo y de su ambiente, consiguientemente a las dificultades de procesar el éxito y la estructuración de la individualidad como característica típica de funcionar con independencia de los demás; los esquemas cognitivos derivados son los de dependencia, vulnerabilidad al daño o la enfermedad, apego o fracaso; el origen de dichos esquemas es resultante en las familias que cuestionan la capacidad del niño o niña para actuar autónomamente o la sobreprotección. El dominio de límites deteriorados hace referencia a las dificultades de establecer límites internos y de responsabilidad a los demás. Los esquemas cognitivos típicos son la grandiosidad y autocontrol insuficiente; en la misma lógica anterior, los orígenes se encuentran en las familias con un estilo de crianza permisivo, indulgente y sin dirección. El cuarto dominio de orientación a los demás, expresado en el énfasis excesivo en los deseos y sentimientos de los demás, deriva en dos esquemas cognitivos: autosacrificio y subyugación; el origen puede deberse a las experiencias que tienen los niños en la aceptación condicionada, que implica que debe suprimir aspectos importantes de sí mismos para que sean admitidas determinadas conductas. El quinto dominio se refiere a la vigilancia excesiva e inhibición, que produce los esquemas cognitivos de inhibición emocional y estándares inalcanzables. El origen es atribuido a las familias severas con un estilo de crianza punitivo. 
En otro momento, Young y Brown (1994) construyeron un cuestionario de esquemas y propusieron el análisis factorial en indicadores de ajuste para identificar quince esquemas cognitivos: abandono/inestabilidad, abuso, privación emocional, imperfección o culpa, aislamiento social, dependencia o incompetencia, vulnerabilidad al daño o a la enfermedad, apego, fracaso, grandiosidad, autocontrol insuficiente, subyugación, autosacrificio, inhibición emocional, estándares inalcanzables. Excepto cuatro ítems, cuyos pesos oscilaban entre .33 y .39 , todos los demás ítems obtuvieron pesos adecuados en las subescalas correspondientes. En cuanto a la consistencia interna de las subescalas, la grandiosidad fue la que obtuvo un coeficiente más bajo (.57), mientras que la mayoría de las subescalas coeficientes mostró entre .72 y .83

Un trabajo original es el realizado por Calvete, Estévez, Landin (2002) sobre autodiálogo y consiste en solicitar a los estudiantes universitarios, recordar situaciones en las que se habían sentido felices, tristes, ansiosos o enfadados, y que escribieran los pensamientos que pasaron por sus mentes. A partir aproximadamente de 500 relatos obtenidos; se seleccionaron las situaciones y el autodiálogo positivo y negativo más frecuentes. En la selección no se incluyeron grandes eventos vitales, tales como la muerte de un familiar, ni aquel autodiálogo que pareciera atípico o peculiar.

En esta línea y con los antecedentes de Young, los investigadores indagaron sobre las relaciones entre tipos de autodiálogo, y encontraron que el patrón de correlaciones entre subescalas y escalas de autodiálogo fue casi idéntico al obtenido en el estudio original de Young. Las dos escalas amplias, de autodiálogo positivo y de autodiálogo negativo, propuesta por Calvete y colaboradores, mostraron ser relativamente independientes entre sí, lo que justifica una vez más la decisión de estudiar de medida de ambas escalas por separado.

Puestas a prueba estas dos escalas de Inventario de Autodiálogo, Calvete Estévez, Landin (2002) obtuvieron coeficientes alpha de .90 y .80 para la escala de autodiálogo negativo (EAN) y positivo (EAP), respectivamente. Asimismo, los coeficientes alpha por subescalas fueron $.82, .83, .87, .73, .74, \mathrm{y} .44$ para pensamiento de ira, pensamientos de depresión, pensamiento de ansiedad, minimización, afecto positivo y autoinstrucciones de afrontamiento, respectivamente. Además en el mencionado estudio, las subescalas se asociaron a los síntomas de depresión, ansiedad e ira, de acuerdo con la teoría de la especificidad cognitiva y el modelo tripartito de la ansiedad y depresión.

Es preciso señalar otros antecedentes sobre los estudios del diálogo, categorizados como autodiálogo interior (Albert Ellis,) y pensamiento automático (Aaron Beck) provenientes de la clínica psicológica referido al lenguaje para sí mismo, en silencio, durante un tiempo relativamente prolongado, que se convierte en una conversación interior continua, que va configurando experiencias diarias, sobre lo "que se va a hacer", "lo que sentimos" o lo que le "está pasando a los demás". También se refiere a planes, comentarios privados o imaginarios, muchos de los cuales la persona los da como ciertos o le crea complicaciones emocionales. Un ejemplo elocuente es el que plantea Knaus (1974) referida a la siguiente experiencia: "una persona se enfadó mucho cuando un desconocido la empujó, pero al observar que el desconocido era ciego sintió tristeza y culpa". En un primer momento igual pensó "a ver si miras donde vas", y después algo como "ipobre hombre, es ciego!". Con este ejemplo se puede observar cómo al cambiar los pensamientos ante una determinada situación, es decir, al modificar la interpretación de los hechos, cambian también los sentimientos. 
De lo anterior se desprende que las personas pueden tener muchos autodiálogos, por consiguiente, los estudiantes universitarios, ante una diversidad de situaciones y trabajo intelectual, con cargas emotivas y afectivas muy intensas, de "éxito" o "fracaso" en una línea progresiva de perfeccionamiento, el autodiálogo es una práctica permanente.

La investigación psicológica ha determinado dos tipos de autodiálogos que configuran sistemas motivacionales (Amsel y Fichten, 1998; Cacioppo, Piel y Ernst, 1997; Fichten, Amsel, Robillard y Tagalakis, 1991, Schwartz, 1997), expresados en pensamientos positivos y negativos, que en las más de las veces se presentan como una dimensión bipolar.

El interés de investigación y de intervención especializada en este tipo de manifestaciones del pensamiento promovió la construcción de instrumentos de evaluación, entre los cuales distinguimos los siguientes: (1) Cuestionario de Pensamientos Automáticos Positivos ATQ-P (Ingram y Winiski, 1988; (2) Evaluación del pensamiento negativo con el Cuestionario de Pensamientos Automáticos ATQ (Hollín y Kendall, 1980); (3) Inclusión en subescalas diferentes como el Inventario de Autodiálogo BST, Burnett, 1996, que contiene dos subescalas: Autodiálogo Positivo y Autodiálogo Negativo, (4) Cuestionario de Autoafirmaciones de Interacción Social SISST de Glass. Merluzzi, Biever y Larsen, 1982, con dos subescalas de Autodiálogo facilitador y autodiálogo inhibitorio.

La multiplicidad de situaciones del pensamiento motivó también que se identifiquen sistemas de clasificación; así Uhlemann y Plater (1990) enfatizaron en autoafirmaciones, a las que agruparon en cinco categorías: (1) pensamientos sobre uno mismo; (2) Pensamientos sobre la interacción; (3) Pensamientos sobre los otros individuos implicados, (4) Pensamientos irrelevantes; y (5) Autodiálogo de clasificación. Por su parte Ronan, Rowe y Kendall (1988) identificaron dos dimensiones: (1) Atención negativa centrada en el propio yo, (2) Autoconcepto positivo y expectativas positivas. Leitenberg, Yost y Carroll-Wilson (1986) clasificaron el autodiálogo en (1) Tremendismo; (2) Sobregeneralización; (3) Personalización y (4) Abstracción selectiva.

Ampliando la información desde la psicología clínica, un tipo especial de investigaciones realizadas para estudiar el autodiálogo a través de las instrucciones de afrontamiento (Calvete, Cardeñoso, 2002; Kendall y Chamsky, 1991; Prins y Hanewald, 1997; Zatz y Chassin, 1985; Meichenbaum, 1977), precisan las verbalizaciones internas que acompañan la actividad de la persona. Como se puede apreciar, el objeto de estudio son los mensajes internos hacia uno mismo que concretan la actividad cognitiva en la conducta humana, o también la verbalización interna que orienta la ejecución de la tarea, en el caso de los universitarios las autoinstrucciones para el aprendizaje.

Desde la psicología educativa, asumimos que el aprendizaje es el hecho privilegiado de la educación, pese a que en la historia de la educación se han realizado muchas conceptualizaciones sobre el aprendizaje. En el presente estudio tomamos los planteamientos del constructivismo pedagógico dominantemente influenciados por el paradigma cognitivo, que establece las relaciones cognitivas específicas entre el aprendizaje y el desarrollo personal del alumno, enfatizando en este último. Se trata de identificar el papel del pensamiento en la elaboración de aprendizajes, aclarando que no destacamos la relación estrecha del individuo, sino en el papel que cumple la actividad cognitiva en la construcción o reconstrucción del conocimiento mediante el aprendizaje. Está claro que el aprendizaje tiene una génesis social, pero al aprendizaje solamente lo podemos entender de manera 
individual; entonces debe existir un proceso por el cual el pensamiento oriente la actividad ejecutora o de tarea.

Para Albert Ellis, la conducta depende más de los sistemas de creencias individuales y de las formas de interpretar las situaciones que de las condiciones objetivas. Porque consideramos importante para nuestro tema, repasemos algunas hipótesis planteadas por Albert Ellis en el $\mathrm{ABC}$ de la terapia racional emotiva: Hipótesis 1. El pensamiento crea la emoción. El pensamiento humano y la emoción no constituyen dos procesos separados o diferentes, sino que se hallan muy relacionados. El conocimiento representa una operación intermediaria entre los estímulos y las respuestas. Lo que llamamos emociones y comportamientos no se originan de las reacciones de las personas y su medio ambiente, sino también de sus pensamientos, creencias y actitudes ante ese ambiente. Hipótesis 2. Los pensamientos semánticos y las autoafirmaciones afectan a la conducta. Las personas siempre se hablan a sí mismas; las diferentes cosas que ellas se dicen y la forma como se las dicen afectan muchísimo a sus emociones y a su conducta y algunas veces las lleva a sentirse perturbadas emocionalmente. Hipótesis 3. Los estados de ánimo dependen de la cognición. Los estados de ánimo de las personas dependen muchísimo de lo que crean o de lo que se digan a sí mismas. Cuando se dicen a sí mismas y creen en ideas optimistas, esperanzadoras y alegres tienden a sentirse felices, jubilosas, contentas o serenas; cuando se dicen o creen en ideas pesimistas, cínicas, desesperadas y predicen un futuro sombrío, tienden a sentirse tristes, malhumoradas, desgraciadas y deprimidas. La terapia eficaz a veces consiste en ayudar a los clientes a tener ideas optimistas y alegres y a dejar sus puntos de vista indebidamente pesimistas acerca del presente y del futuro. Hipótesis 4. La conciencia, la "insight" y la autoindicación afectan a la conducta. Las personas no solamente tienen la capacidad de pensar (y generalizar) sino de pensar acerca de sus pensamientos y de pensar acerca del pensamiento de sus pensamientos. Casi siempre observan y conocen su conducta y por esta observación y cognición cambian mucho su conducta. Siempre que se sientan perturbadas emocionalmente (v. g. con ansiedad, deprimidas u hostiles), tienden a percibir y pensar acerca de su perturbación y por ello, o se perturban más (v.g. angustiadas por su ansiedad o deprimidas por sus sentimientos de depresión) o disminuyen su perturbación. La conciencia, la insight y la autoindicación, por lo tanto incluye los procesos cognitivos que afectan muchísimo a la conducta y al cambio de conducta.

Las investigaciones sobre la depresión entre hombres y mujeres señalaron que las mujeres son más propensas a sufrir de este cuadro clínico en el periodo climaterio, mientras son los hombres que la padecen más en la juventud. En nuestro país (2007) el Instituto Nacional de Salud Mental Honorio Delgado-Hideyo Noguchi da como resultado que las mujeres tienen mayor propensión a padecer de este estado con un $23.3 \%$ a diferencia de los hombres que sería un $14.5 \%$. También se observa que los niveles de ansiedad son mayores en mujeres que en hombres; en estas se presentan en un $25.3 \%$ mientras que en los hombres sería en un $16.6 \%$; una interpretación podría ser que las mujeres tendrían mayores posibilidades a contraer estos cuadros debido al nivel de tensión que tienen durante su vida rutinaria; los hombres por el contrario se muestran más reacios, quizá son más resilentes a los problemas.

Los estudios cognitivos de la depresión, los revisamos desde la perspectiva de dos clínicos que han contribuido notablemente en los modelos de tratamiento antidepresivos. Nos referimos a Aaron Beck $(1955,1995)$ y Albert Ellis (1975). Beck (1995) estudia las 
cogniciones negativas de sí mismo y de su entorno, y desde la clínica inicia una estrategia para elaborar técnicas que aporten una labor superadora de las cogniciones.

Otra referencia es la teoría de los constructos personales (George Kelly, 1955) que sostiene que las personas construyen estructuras que se adelantan a los sucesos, es decir, las personas manejan las expectativas y las consecuencias derivadas.

Es preciso señalar que otros autores han establecido una vinculación más directa con los procesos cognitivos y afectivos de manera independiente y articulados entre sí. Revisamos a continuación algunas expresiones. Keefe (1988) conceptúa a los estilos de aprendizaje como rasgos cognitivos, afectivos y fisiológicos, que sirven como indicadores relativamente estables, de cómo el aprendiz percibe, interacciona y responde a sus ambientes de aprendizaje. Kolb (1984) emplea el concepto de estilo de aprendizaje dentro de la experiencia, en los siguientes términos "algunas capacidades de aprender se destacan por encima de otras como resultado del aparato hereditario de las experiencias vitales propias, y de las exigencias del medio ambiente actual; llegamos a resolver de manera característica, los conflictos entre el ser activo y reflexivo y entre el ser inmediato y analítico; algunas personas desarrollan mentes que sobresalen en la conversión de hechos dispares en teorías coherentes y, sin embargo, estas mismas personas son incapaces de deducir hipótesis a partir de su teoría, o no se interesan por hacerlo; otras personas son genios lógicos, pero encuentran imposible sumergirse en una experiencia y entregarse a ella.

Del estudio cognitivo del aprendizaje, seleccionamos la categorización de "estilo de aprendizaje" abordado como "la intervención de la mente en el tratamiento de la información" o lo que es lo mismo "cómo el aprendizaje es influido por las percepciones de cada individuo" (Messick, 1969, Coop y Brown, 1978, Hill, 1971, Witkin, 1975). Para Scheck, un estilo de aprendizaje "es simplemente el estilo cognitivo que un individuo manifiesta cuando se confronta con una tarea de aprendizaje". Butler (1982) indica que los estilos de aprendizaje "señalan el significado natural por el que una persona más fácil, efectiva y eficientemente se comprende a sí misma, el mundo y las relaciones entre ambos”. Smith (1988) sostiene que los estilos de aprendizaje "son modelos característicos por los que un individuo procesa la información, siente y se comporta en situaciones de aprendizaje".

Queremos resaltar que la individuación del aprendizaje no aísla al individuo de otros ámbitos y componentes, simplemente precisa que el sujeto aprende por sí mismo, mediado por su cognición y en nuestro caso influenciado hipotéticamente por el autodiálogo. Smith (1988) realiza un inventario de las condiciones para que una persona se encuentre en la empresa de aprender, éstas son las siguientes: (1) Cómo controlar el propio aprendizaje; (2) Cómo desarrollar un plan personal de aprendizaje; (3) Cómo diagnosticar sus puntos fuertes y debilidades como discente; (4) Cómo describir su estilo de aprendizaje; (5) Cómo superar los bloqueos personales de aprendizaje; (6) En qué condiciones aprende mejor; (7) Cómo aprender de la experiencia de cada día; (8) Cómo aprender de la radio, TV, prensa, computadoras; (9) Cómo participar en grupos de discusión y de resolución de problemas; (10) Cómo aprovechar al máximo una conferencia o un curso; (11) Cómo aprender de un tutor; (12) Cómo usar la intuición para el aprendizaje.

Catalina Alonso, Domingo Gallego y Meter Horney $(1992,2005)$ plantean una propuesta sobre cuatro estilos de aprendizaje: estilo activo, estilo reflexivo, estilo teórico y estilo pragmático. Estos involucran una cantidad de características, seleccionadas y resultantes de 
análisis estadísticos muy finos que se han realizado en estudiantes universitarios, divididos entre características principales y otras secundarias. A continuación solamente señalamos las primeras: características principales del estilo activo: animador, improvisador, descubridor, arriesgado y espontáneo; características principales del estilo reflexivo: ponderado, concienzudo, receptivo, analítico y exhaustivo; características principales del estilo teórico: metódico, lógico, objetivo, crítico y estructurado; características principales del estilo pragmático: experimentador, práctico, directo, eficaz y realista.

Alonso y colaboradores (1991) realizaron una investigación sobre estilos de aprendizaje en estudiantes de las Universidades Complutense y Politécnica de Madrid, traduciendo el cuestionario original y sometiéndolo a un piloto para su adaptación al sistema semántico español y al criterio de jueces, denominando CHAEA. Con él se construyeron perfiles de estilos de aprendizaje con las experiencias vitales y las reflexiones expresadas por los alumnos. Se realizó el análisis estadístico correspondiente utilizando el coeficiente Alfa de Cronbach para medir la consistencia interna de la escala (prueba de fiabilidad), obteniendo los siguientes resultados: estilo activo .6272; estilo reflexivo .7275; estilo teórico .6584 y estilo pragmático .5854 . En cuanto a la validez del cuestionario se realizaron varios análisis, como los siguientes: análisis de contenido; análisis de ítems; análisis factorial del total de ítems; análisis factorial de cada uno de los cuatro factores; y análisis factorial de los cuatro estilos de aprendizaje a partir de las medias totales de cada factor. La investigación concluyó con un Baremo de Interpretación, que facilita la interpretación de puntuaciones.

Conceptuando los estilos de aprendizaje como las distintas formas que emplean las personas para aprender, el cómo o la pericia empleada. Stenberg (1999) diferencia el estilo y la actitud; el estilo es la forma preferida para utilizar la actitud. En otra elaboración, Schmeck (1988) señala que un estilo de aprendizaje es la predisposición del sujeto para adoptar una estrategia particular de aprendizaje con independencia de las demandas específicas de la tarea, refrendando una consistencia estable en la forma de atender, percibir y pensar en la aplicación de la estrategia de aprendizaje. Schmeck identifica cuatro estilos de aprendizaje: profundo, elaborativo, retención de hechos y metódico.

El problema de investigación se plantea en los siguientes términos ¿Cómo se asocian los esquemas de autodiálogo, positivos y negativos, y estilos de aprendizaje en los estudiantes universitarios de distintas áreas académicas de la Universidad Nacional Mayor de San Marcos?

\section{MÉTODO}

\section{Diseño de la investigación}

La investigación es de carácter descriptivo, comparativo y correlacional, es decir, que se realizará la medición de las variables involucradas y los resultados de la medición serán comparados entre sí por el grado de aproximación en cada una de las áreas académicas.

\section{Población y muestra}

La población corresponde a los estudiantes de la Universidad Nacional Mayor de San Marcos matriculados en 20 facultades y 56 Escuelas Académico-Profesionales. 
La población estudiantil suma unos 30,000, y un hecho relevante es que en los últimos años la tendencia de aumento de ingreso es de estudiantes mujeres (55\%) frente a un $45 \%$ de hombres; además el $52 \%$ proviene de la educación pública y un $48 \%$ de la educación privada.

Las áreas académicas que comprenden los estudios en la Universidad Nacional Mayor de San Marcos son las siguientes: Área A. Ciencias de la Salud (Medicina Humana, Obstetricia, Enfermería, Tecnología Médica, Nutrición, Farmacia y Bioquímica, Odontología, Medicina Veterinaria y Psicología); Área B. Humanidades (Derecho, Ciencia Política, Literatura, Filosofía, Lingüística, Comunicación Social, Arte, Bibliotecología y Ciencias de la Información, Educación Inicial, Educación Primaria, Educación Secundaria, Educación Física, Historia, Sociología, Antropología, Arqueología, Trabajo social, Geografía; Área C. Ciencias Básicas (Química, Ciencias Biológicas, Genética y Biotecnología, Microbiología y Parasitología, Física, Matemáticas, Investigación Operativa, Computación Científica); Área D. Ingenierías (Ingeniería Química, Ingeniería Mecánica de Fluidos, Ingeniería Geológica, Ingeniería Geográfica, Ingeniería de Minas, Ingeniería Metalúrgica, Ingeniería Industrial, Ingeniería Electrónica, Ingeniería Eléctrica, Ingeniería de Sistemas): Área E. Económico Empresariales (Administración, Administración de Turismo, Administración de Negocios Internacionales, Contabilidad y Economía).

Tabla N. ${ }^{0}$ 1. Distribución de la muestra por áreas académicas y género.

\begin{tabular}{clcccc}
\hline & & \multicolumn{2}{c}{ Sexo } & Total \\
\hline $\begin{array}{c}\text { Área } \\
\text { académica }\end{array}$ & Ciencias de la Salud & $\mathrm{N}$ & 27 & 69 & 96 \\
& & $\%$ & $28.1 \%$ & $71.9 \%$ & $100.0 \%$ \\
& & & Hombre & Mujer \\
& Ciencias básicas & $\mathrm{N}$ & 25 & 19 & 44 \\
& & $\%$ & $56.8 \%$ & $43.2 \%$ & $100.0 \%$ \\
& Ingenierías & $\mathrm{N}$ & 42 & 25 & 67 \\
& & $\%$ & $62.7 \%$ & $37.3 \%$ & $100.0 \%$ \\
& Ciencias empresariales & $\mathrm{N}$ & 29 & 23 & 52 \\
& & $\%$ & $55.8 \%$ & $44.2 \%$ & $100.0 \%$ \\
& Humanidades & $\mathrm{N}$ & 10 & 23 & 33 \\
& & $\%$ & $30.3 \%$ & $69.7 \%$ & $100.0 \%$ \\
& Total & $\mathrm{N}$ & 133 & 159 & 292 \\
& & & $45.5 \%$ & $54.5 \%$ & $100.0 \%$ \\
\hline
\end{tabular}

La muestra ha sido de carácter probabilístico estratificado, constituida por 292 estudiantes (Tabla N. ${ }^{\circ}$ 1), distribuidos de la siguiente manera: Área de Ciencias de la Salud: 96, Área de Ciencias Básicas: 44; Área de Ingenierías: 67; Área de Ciencias Empresariales: 52 y Área de Humanidades: 33. 
Tabla N. 2. Distribución de la muestra por edad y género.

\begin{tabular}{|c|c|c|c|c|c|}
\hline & & & \multicolumn{2}{|c|}{ Sexo } & \multirow{2}{*}{$\begin{array}{c}\text { Total } \\
\text { Hombre }\end{array}$} \\
\hline & & & Hombre & Mujer & \\
\hline \multirow{10}{*}{ Edad } & \multirow{2}{*}{19} & $\mathrm{~N}$ & 14 & 23 & 37 \\
\hline & & $\%$ & $37.8 \%$ & $62.2 \%$ & $100.0 \%$ \\
\hline & \multirow{2}{*}{20} & $\mathrm{~N}$ & 37 & 47 & 84 \\
\hline & & $\%$ & $44.0 \%$ & $56.0 \%$ & $100.0 \%$ \\
\hline & \multirow{2}{*}{21} & $\mathrm{~N}$ & 46 & 63 & 109 \\
\hline & & $\%$ & $42.2 \%$ & $57.8 \%$ & $100.0 \%$ \\
\hline & \multirow{2}{*}{22} & $\mathrm{~N}$ & 36 & 23 & 59 \\
\hline & & $\%$ & $61.0 \%$ & $39.0 \%$ & $100.0 \%$ \\
\hline & \multirow{2}{*}{23} & $\mathrm{~N}$ & 0 & 3 & 3 \\
\hline & & $\%$ & $.0 \%$ & $100.0 \%$ & $100.0 \%$ \\
\hline \multirow{2}{*}{\multicolumn{2}{|c|}{ Total }} & $\mathrm{N}$ & 133 & 159 & 292 \\
\hline & & $\%$ & $45.5 \%$ & $54.5 \%$ & $100.0 \%$ \\
\hline
\end{tabular}

La edad promedio de los estudiantes de la muestra investigada es de 20 años, con un intervalo entre los 19 y 23 (Tabla N. ${ }^{\circ} 3$ ).

Tabla N. ${ }^{\circ}$ 3. Estadística de la muestra por edad.

\begin{tabular}{lc}
\hline Media & 20.68 \\
Mediana & 21 \\
Moda & 21 \\
Desv. típ. & .969 \\
Mínimo & 19 \\
Máximo & 23 \\
\hline
\end{tabular}

\section{INSTRUMENTOS}

\section{Instrumentos de Investigación}

El Inventario de autodiálogo para jóvenes (Calvete, Estévez, Landin).Consta de dos escalas amplias, uno de autodiálogo positivo y otro de autodiálogo negativo. En ambas escalas se solicita a los participantes que indiquen con qué probabilidad se "dirían a sí mismos" cada una de 52 afirmaciones en respuesta a diez situaciones imaginarias, empleando una escala de respuestas de cuatro puntos $(1=$ no muy probable; $2=$ algo probable; 3 = bastante probable y $4=$ muy probable). Como se sabe los ítems fueron obtenidos a partir de relatos de un grupo de 110 universitarios y empleando un procedimiento de listado de pensamiento 
Cuestionario Honey-Alonso de Estilos de Aprendizaje (CHAEA) adaptado en España del original Cuestionario de Estilos de Aprendizaje LSQ Learning Styles Questionnaire, de Honey de Reino Unido. El CHAEA tiene la siguiente estructura en su presentación y resultados; datos personales socioacadémicos, instrucciones de realización, nómina de ítems sobre estilos de aprendizaje y espacio para respuestas y perfil de aprendizaje numérico y gráfico.

\section{Variables relacionadas}

- Autodiálogo positivo (minimización, autoinstrucciones de afrontamiento y afecto positivo; y autodiálogo negativo (depresión, ansiedad, ira).

- Estilos de aprendizaje: Activo, reflexivo, teórico y pragmático.

- Áreas académicas donde se encuentra matriculado el estudiante: Salud, Ciencias Básicas, Ingenierías, Empresarial, y Humanidades.

\section{Variables controladas}

- Género.

- Edad.

\section{Hipótesis}

Existen diferencias en las asociaciones de los esquemas de autodiálogo positivo y negativo y los estilos de aprendizaje, en los estudiantes, según las áreas académicas de los estudiantes sanmarquinos.

\section{RESULTADOS}

El análisis de consistencia se aplicó para evaluar el grado en que los ítems de los instrumentos empleados se encuentran relacionados entre sí, hallando que mediante el coeficiente Alfa de Cronbach para la subescala del autodiálogo positivo, la decisión es aceptable, mientras que para el autodiálogo negativo es alta; de otro lado, una vez desagregados los estilos de aprendizaje, en las subescalas correspondientes, encontramos que para el estilo activo es buena y para los estilos reflexivo, teórico y pragmático resultaron altas. 
Tabla N. ${ }^{0}$ 4. Coeficientes de confiabilidad del cuestionario de inventario de autodiálogo y de los estilos de aprendizaje de Honey-Alonso mediante el método de la consistencia interna.

\begin{tabular}{lccc}
\hline & Alfa de Cronbach & Número de ítems & Decisión \\
\hline Autodiálogo positivo & 0.597 & 26 & Aceptable \\
Autodiálogo negativo & 0.937 & 26 & Alta \\
Estilo activo & 0.726 & 20 & Buena \\
Estilo reflexivo & 0.834 & 20 & Alta \\
Estilo teórico & 0.832 & 20 & Alta \\
Estilo pragmático & 0.801 & 20 & Alta \\
\hline
\end{tabular}

De acuerdo al análisis de correspondencias múltiples, se ha identificado las relaciones entre variables y similitudes o diferencias entre categorías de las variables y entre las variables mismas, precisando que el análisis de correspondencias se calculó la matriz de correlaciones con el objetivo de seleccionar cuales eran las variables a considerar para el análisis (se consideraron las variables que tenían correlaciones superior a 0.5 o inferiores a -0.5). El análisis se ha realizado para cada área académica, de las cinco áreas del análisis realizado se halló lo siguiente: para el área de Salud un 60\% de varianza (variabilidad) explicada con dos dimensiones (en las dimensiones se asocian los perfiles), luego sigue el área de Ingenierías con un $40 \%$ de varianza explicada y las áreas de Humanidades, Empresariales y Ciencias Básicas con un $30 \%$ de varianza explicada. Todas ellas también explicadas con dos dimensiones. Es importante resaltar que en los mapas de correspondencias se identifican solo las agrupaciones o perfiles que están alejados del origen.

Tabla N. ${ }^{0}$ 5. Análisis estadísticos de los instrumentos de autodiálogo y de estilos de aprendizaje.

\begin{tabular}{lcc}
\hline & Media & Desv. típ. \\
\cline { 2 - 3 } Depresión & 12.72 & 7.488 \\
Ansiedad & 9.84 & 5.358 \\
Ira & 6.84 & 4.490 \\
Autodiálogo negativo & 29.40 & 15.13 \\
Minimización & 17.19 & 4.562 \\
Autoinstrucciones & 10.87 & 2.236 \\
Afecto positivo & 17.58 & 3.961 \\
Autodiálogo positivo & 45.63 & 7.97 \\
Estado mental SOM & .6233 & .13427 \\
Estilo activo & 11.71 & 3.794 \\
Estilo reflexivo & 9.76 & 4.821 \\
Estilo teórico & 9.84 & 5.032 \\
Estilo pragmático & 8.88 & 4.504 \\
\hline
\end{tabular}




\section{Área de Ciencias Básicas}

$\mathrm{Al}$ observar la matriz de correlaciones (Tabla N. ${ }^{\circ} 7$ ) resalta la relación inversa (correlación -0.869) existente entre (estilo 12 y estilo 36), es decir, mientras más reflexivo se comporta el estudiante, menos pragmático se comportará y viceversa.

$\mathrm{Al}$ aplicar el análisis de correspondencias respecto de la Figura N. ${ }^{\circ} 1$ de las medidas de discriminación, resaltan las relaciones entre (auto 4, 7 y 30), entre (estilo 12 y 22) y entre (estilo 36 y 59); observando el mapa de correspondencias (Figura N. ${ }^{\circ}$ 6) podemos ver agrupaciones (similitudes) entre categorías, por un lado, el pensar muy probable (auto 8), bastante probable (auto10), algo probable (auto 7, 32, 46, 48), nada probable (auto 29), actuar en desacuerdo (estilo 40) y, por otro lado, actuar en desacuerdo (estilo 12 y 53), de acuerdo (estilo 36) ambas agrupaciones asociadas a la dimensión 1 y se oponen en esta dimensión, otra agrupación asociada a la dimensión 2 entre pensar nada probable (auto 32,20 ), bastante probable (auto 8, 44), algo probable (auto 42), muy probable (auto 4), actuar de acuerdo (estilo 35, 59).

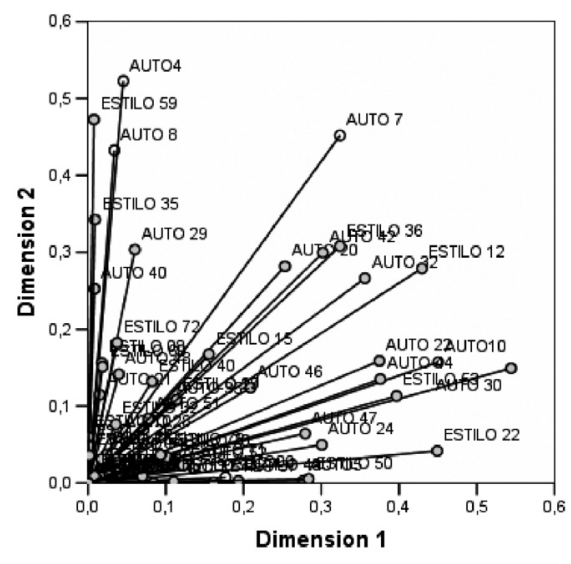

Figura N. ${ }^{0}$ 1. Medidas de discriminación en el área de Ciencias Básicas.

\section{Área Económico-Empresarial}

Se puede apreciar una relación inversa (Tabla N. ${ }^{\circ}$ 8) (correlación -0.711) entre (auto 3 y auto 9), es decir, en cuanto más piensa negativamente el estudiante de empresariales, pensará positivamente menos.

Del análisis de correspondencias (Figura N. ${ }^{\circ}$ 2), de medidas de discriminación nos muestra las siguientes relaciones; entre (auto 3, 9, 30, 44), entre (auto 15 y 16) y entre (auto 29 y estilo 71).

En el mapa de correspondencias (Figura N. ${ }^{\circ}$ 7) podemos identificar las siguientes agrupaciones entre categorías (perfiles); por un lado, pensar nada probable (auto 6 y 46), pensar bastante probable (auto 44) asociada a la dimensión $1 \mathrm{y}$, por otro lado, pensar nada probable (auto 1 y 16), algo probable (auto 36), bastante probable (auto 6), actuar de acuerdo (estilo 5 y 57) asociada a la dimensión 2. 


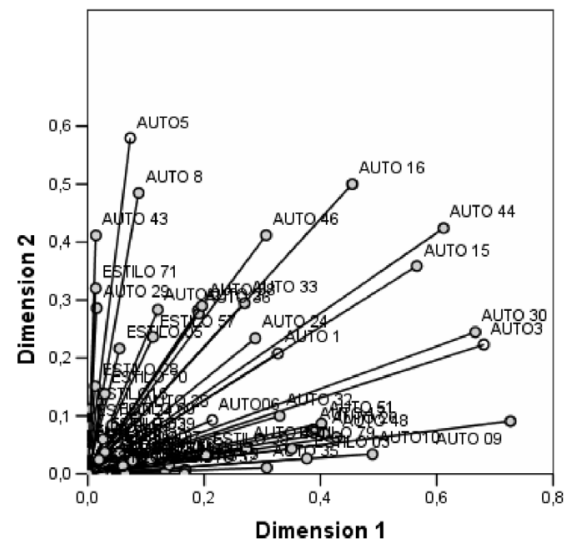

Figura N. ${ }^{0}$ 2. Medida de discriminación en el área Económico-Empresariales.

\section{Área de Humanidades}

De la matriz de correlaciones (Tabla N. ${ }^{\circ}$ 9), se distingue la relación directa entre los estilos 16 y 32 (correlación 0.635) y la relación inversa (correlación -0.63) entre los estilos 12 y 44, que al igual que en los estudiantes de Ciencias Básicas se observa que mientras más reflexivo se comporta el estudiante, menos pragmático se comportará y viceversa.

Se pueden identificar más relaciones (Figura N. ${ }^{\circ} 3$ ) de medidas de discriminación, entre (auto 4 y 52) dos pensamientos positivos y (auto 10, 32 y estilo 17) relación entre dos pensamientos, uno negativo y uno positivo, y una actitud teórica.

Del mapa de correspondencias (Figura N. ${ }^{\circ} 8$ ) podemos identificar las siguientes asociaciones por categorías, una agrupación (o perfil) entre pensar bastante probable (auto 6), algo probable (auto 9 y 10), actuar en desacuerdo (estilo 65), actuar de acuerdo (estilo 17), otra agrupación (o perfil) entre pensar bastante probable (auto 17), muy probable (auto 32) ambas asociadas a la dimensión 1, otra agrupación entre pensar algo probable (auto $4,50,51$ ) asociada a la dimensión 2.

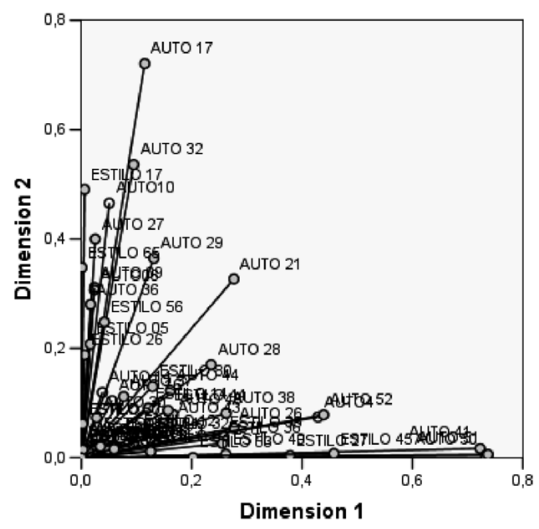

Figura $N^{0} .3$. Medida de discriminación en el área de Humanidades. 


\section{Área de Ingenierías}

De la matriz de correlaciones (Tabla N. ${ }^{\circ} 10$ ), se identifica la relación inversa entre los estilos 35 y 71 (correlación de -0.615), esto quiere decir que el estudiante de ingeniería mientras más activo se comporte menos teórico se comportará y viceversa.

En la Figura N. ${ }^{\circ} 4$, de las medidas de discriminación, podemos observar relaciones importantes entre (auto 4, 14, 30) y entre (auto 48, estilo 60 y estilo 75).

En el mapa de correspondencias (Figura N. ${ }^{\circ} 9$ ), podemos apreciar las agrupaciones por categorías, por un lado un perfil caracterizado por pensar nada probable (auto 2, 20), algo probable (auto 30), muy probable (auto 48) actuar en desacuerdo (estilo 60) y otro perfil caracterizado por pensar nada probable (auto 14 y 15).

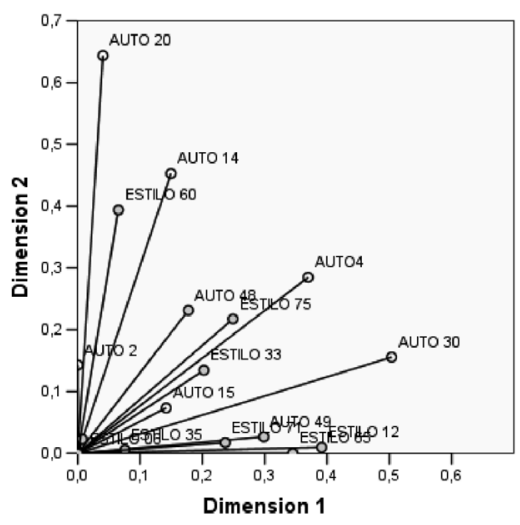

Figura N. ${ }^{0}$ 4. Medida de discriminación en el área de Ingenierías.

\section{Área de Ciencias de la Salud}

Resalta la relación directa entre el estilo 3 y auto 52; (correlación 0.62) (Tabla N. ${ }^{\circ}$ 11), es decir, el estudiante de ciencias de la salud mientras más activo se comporte, más positivo pensará y viceversa.

De la Figura N. ${ }^{\circ}$ 5, de medidas de discriminación, se vuelve a apreciar la relación entre (auto 52 y estilo 3), entre (auto 3 y 46) un pensamiento negativo y uno positivo, entre (estilo 13, 48, 53) dos actitudes activas y una pragmática.

En el mapa de correspondencias (Figura N. ${ }^{\circ} 10$ ), resaltan los siguientes perfiles; uno caracterizado por actuar en desacuerdo (estilo 3, 48), de acuerdo (estilo 53) y otro caracterizado por pensar nada y algo probable auto 52 y nada probable 31 . 


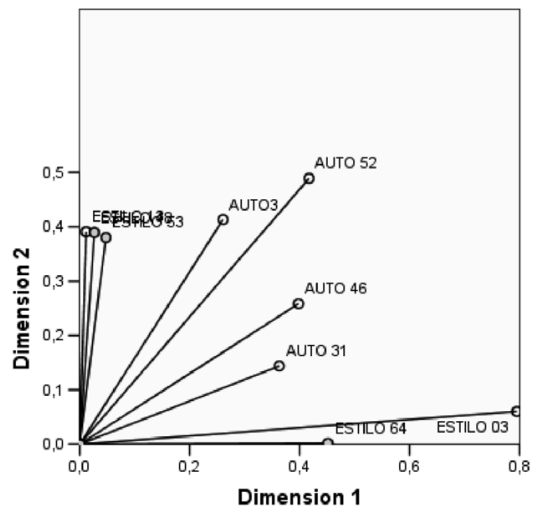

Figura N. ${ }^{0}$ 5. Medida de discriminación en el área de Salud.

\section{DISCUSIÓN}

Tenemos que afirmar que en los resultados obtenidos por el análisis de consistencia, la confiabilidad de los instrumentos empleados, en su mayoría tienen índices altos, como se puede apreciar en la Tabla N. ${ }^{\circ} 4$, por lo tanto, los resultados que analizamos a continuación están respaldados por las exigencias técnicas estadísticas y a nuestro criterio, las inferencias teóricas que se derivan.

De acuerdo a la hipótesis, nos planteamos encontrar las diferencias en las correlaciones de las variables implicadas en los estudiantes de las áreas académicas, con dicho objetivo analizaremos los resultados por área investigada.

Iniciamos nuestro análisis con los resultados de los estudiantes en el área de ciencias básica; aquí encontramos una cadena de hallazgos, siendo los más evidentes, la correlación inversa del estilo de aprendizaje pragmático con el estilo de aprendizaje reflexivo, es decir, una valorización destacada en la aplicación de los conocimientos, contrapuesta con la valorización de profundizar las ideas antes de acatarlas, tal como se presenta en los ítems 12 y 36, referidos al estilo pragmático y reflexivo respectivamente; reforzado el estilo pragmático por la relación entre los ítems 36 y 59; asimismo, se encuentra en la cercanía del ítem 22 y el ítem 12 en el análisis de correspondencias. Del mismo modo podemos identificar los hallazgos de autodiálogo, que de acuerdo al análisis de correspondencia, destacan los ítems 4, 7 y 30, de los cuales dos corresponde al autodiálogo positivo (minimización) y uno al autodiálogo negativo (depresión); es decir que el predominio de la minimización, permite a los estudiantes desarrollar un pensamiento apropiado que conduce a una buena valorización cognitiva de las situaciones, neutralizando a las ideas negativas o tóxicas en que podrían caer sus cogniciones.

En las agrupaciones, los estudiantes de las áreas básicas expresan una variedad de expresiones, así tenemos que por similitudes, de pensar muy probable, bastante probable y algo probable; los hallazgos más relevantes se ubican en los autodiálogos, distribuidos homogéneamente entre autodiálogos positivos y negativos, en el caso de muy probable entre la ansiedad y la minimización y algo probable, depresión y ansiedad, contrapuesto poderosamente con el afecto positivo. 
En cuanto se refiere a los estudiantes del área Económico-empresarial, destaca la relación inversa, entre el mismo autodiálogo negativo (depresión y ansiedad), esto quiere decir que el pensamiento de desvalorización de un hecho se opone al pensamiento de tensión que le produce, relativizándolo con cierta justificación o manejo autorregulado. En un segundo nivel, se hallan agrupadas las relaciones de autodiálogo negativo y positivo, predominando las primeras, es decir, la ansiedad (ítems 9 y 44) y depresión (ítem 3) sobre el autodiálogo positivo (minimización) del ítem 30. En esta misma línea observamos un tercer grupo de relaciones, donde el autodiálogo positivo (minimización) tiene una buena expresión (ítem 15 y 16). Finalmente solo aparece una relación de autodiálogo negativo (ítem 29) con el estilo de aprendizaje teórico (ítem 71).

En los estudiantes del área de Humanidades, los hallazgos tienen una configuración muy peculiar, así tenemos que destaca la relación directa de los estilos reflexivos (ítems 16 y 32) "escucho con más frecuencia que hablo" y "prefiero contar con el mayor número de fuentes de información. "Cuantos más datos reúno para reflexionar, mejor"; y en una relación inversa los estilos pragmáticos (ítem 12) y reflexivo (ítem 44). En cuanto se refiere al autodiálogo, sobresale la minimización (ítem 4) y afecto positivo (ítem 52); y en el cuarto grupo de relaciones dos tipos de pensamientos, uno positivo (ítem 10, minimización) y negativo (ítem 32, ansiedad), y estilo teórico (ítem 17).

En los estudiantes del área de Ingenierías se encuentra una relación inversa en los estilos de aprendizaje activo (ítem 35) y teórico (Item 71); es decir, la disposición hacia el afrontamiento real y efectivo antes que buscar el fundamento o los principios del funcionamiento de los objetos. Es muy destacable el hallazgo de relaciones de autodiálogo positivo, específicamente minimización, como una capacidad para poder manejar las situaciones acertadamente, evitando los pensamientos negativos. Finalmente, un tercer tipo de relaciones, entre el autodiálogo y los estilos teórico y pragmático.

En los estudiantes de Ciencias de la Salud, destaca la relación entre el estilo de aprendizaje activo (ítem 3) y el autodiálogo de afecto positivo (ítem 52); es decir que la actividad se encuentra asociada al pensar positivo de los acontecimientos; surgiendo una paradoja entre un pensamiento depresivo (ítem 3) y un afecto positivo (ítem 46).

Del análisis global de los resultados se puede determinar una asociación dispar de las variables para cada una de las áreas académicas, por lo tanto, concluimos con la aceptación de la hipótesis planteada para la investigación.

\section{CONCLUSIONES}

1. Por el análisis de consistencia, correlación y mapa de correspondencias, los instrumentos empleados posibilita demostrar la pertinencia para una aproximación al conocimiento de cómo se expresa el autodiálogo positivo y negativo y los estilos de aprendizaje en estudiantes universitarios, particularmente en la población investigada.

2. Sin embargo, es preciso indicar que los procesos cognitivos del autodiálogo y los estilos de aprendizaje son procesos independientes; es decir que no se encuentra una asociación directa entre ambos, sino que cada uno tiene sus manifestaciones específicas en los estudiantes de cada área académica.

3. La edad y el género tampoco se encuentran asociadas a un tipo específico de autodiálogo o estilo de aprendizaje 
4. Existen manifestaciones diferentes en las asociaciones de los autodiálogos, positivas y negativas, y los estilos de aprendizaje, en las cinco áreas académicas de la población investigada.

5. En el área de las Ciencias Básicas existen asociaciones inversas entre el estilo de aprendizaje pragmático y el estilo de aprendizaje reflexivo, pero predomina el autodiálogo positivo: minimización.

6. En el área Económico-Empresarial, existe una asociación inversa en el autodiálogo negativo, referida específicamente a la depresión y a la ansiedad; paradójicamente en un segundo nivel, la ansiedad y la depresión pueden regularse recíprocamente, destacando diferentemente una asociación entre el autodiálogo negativo, ansiedad, con el estilo de aprendizaje teórico.

7. En el área de Humanidades existe una relación directa del estilo de aprendizaje reflexivo, aunque hay una tendencia a tener una relación inversa al estilo de aprendizaje pragmático.

8. En el área de Ingenierías existe una relación inversa del estilo de aprendizaje activo con el estilo de aprendizaje teórico.

9. En el área de Ciencias de la Salud existe una relación entre el estilo de aprendizaje activo y el autodiálogo de afecto positivo.

\section{NOTA DE RECONOCIIMIENTO}

El responsable reconoce a los siguientes investigadores: Leoncio Solórzano A., José Luis Perea R., Julio Alvites R., Paúl Murguía B. y Carlos Arroyo P., por compartir la autoría del presente estudio de investigación.

\section{REFERENCIAS BIBLIOGRÁFICAS}

1. Alonso, C. M.; Gallego, D. J. y Honey, P. (1994). Estilos de aprendizaje. Qué son. Cómo se diagnostican. Bilbao: Mensajero.

2. Corveleyn, J. (1996). Histeria y neurosis obsesiva Lima: UL.

3. Estévez A., Calvete E. (2007). Esquemas cognitivos en personas con conducta de juego patológico y su relación con experiencias de crianza. Clínica y Salud 18(1): 23-43. Extraído el 13 de diciembre de 2009 desde http://scielo.isciii.es/scielo. php?script $=$ sci_arttext\&pid $=$ S1130-52742007000100003\&lng $=$ es.A $<$ aaaaaaa AA

4. García, E. y Pascual, F. (1994). Estilos de aprendizaje y cognitivos. En A. Puente (ed.). Estilos de aprendizaje y enseñanza. Madrid: CEPE.

5. Delgado, A. (2004). Relación entre estilos de aprendizaje y los estilos de pensamiento en estudiantes de maestría considerando las especialidades profesionales y el tipo de universidad. Tesis de Doctor en Psicología. Lima: UNMSM.

6. Escurra, M. M. (1991). Adaptación del inventario de estilos de aprendizaje de Kolb en estudiantes de psicología pertenecientes a diferentes universidades de Lima Metropolitana. Tesis de Licenciatura. Lima: UNMSM. 
7. Labatut P., E. (2005). Evaluación de los estilos de aprendizaje y metacognición en estudiantes universitarios. En Revista de Psicopedagogía $N^{o} 67$

8. Oertony, A., Clore, G. y Cikkuns, A. (1988). La estructura cognitiva de las emociones. Madrid: Siglo XXI.

9. Sarason, I. y Sarason, B. (1988). Psicología anormal. Los problemas de la conducta desadaptada. México: Trillas

10. Sotelo, L. y Sotelo, N. (1999). Relación entre estilos de aprendizaje y rendimiento académico en estudiantes de 4to y 5to de secundaria de colegios estatales de Lima Metropolitana. Tesis de Magíster en Psicología. Lima: UNIFE

11. Van Dijk, Teun (1999). Ideología Barcelona: Gedisa.

12. Zavala G., H. (2008). Relación entre el estilo de aprendizaje y el nivel de comprensión lectora en estudiantes de quinto de secundaria de colegios estatales y particulares de Lima Metropolitana. Tesis de Magíster.Lima: UNMSM.

\section{ANEXOS}
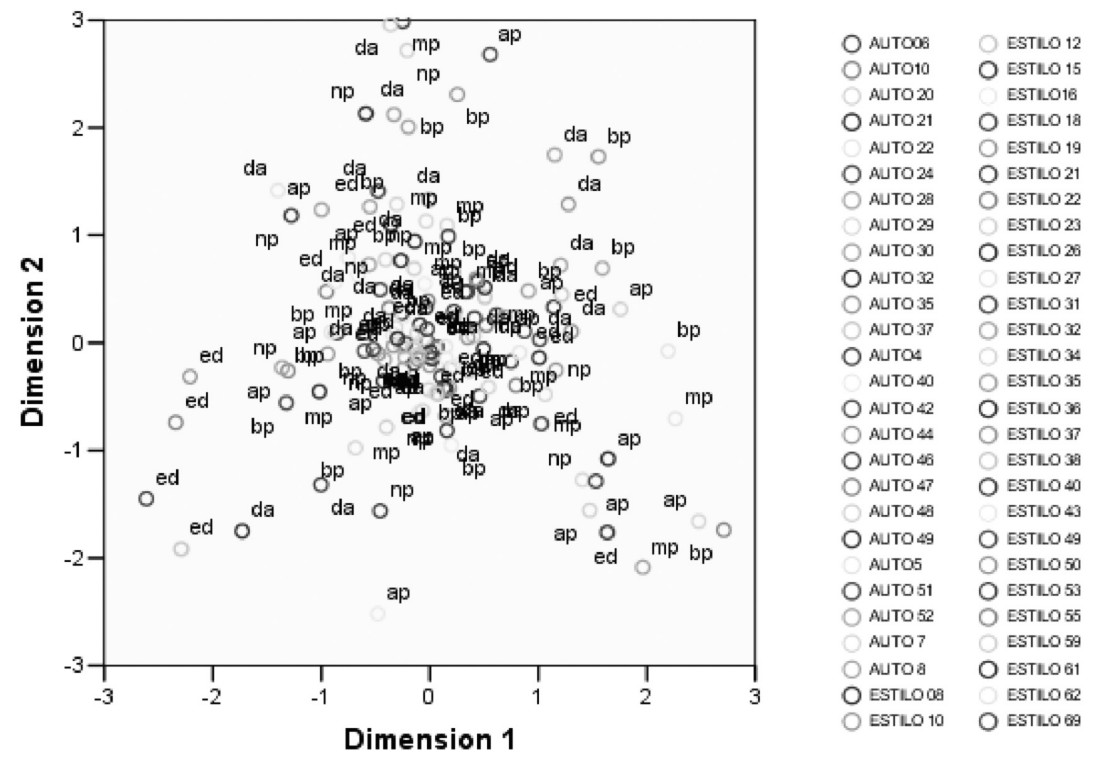

Figura N. ${ }^{0}$ 6. Mapa de correspondencias del área de Ciencias Básicas. 

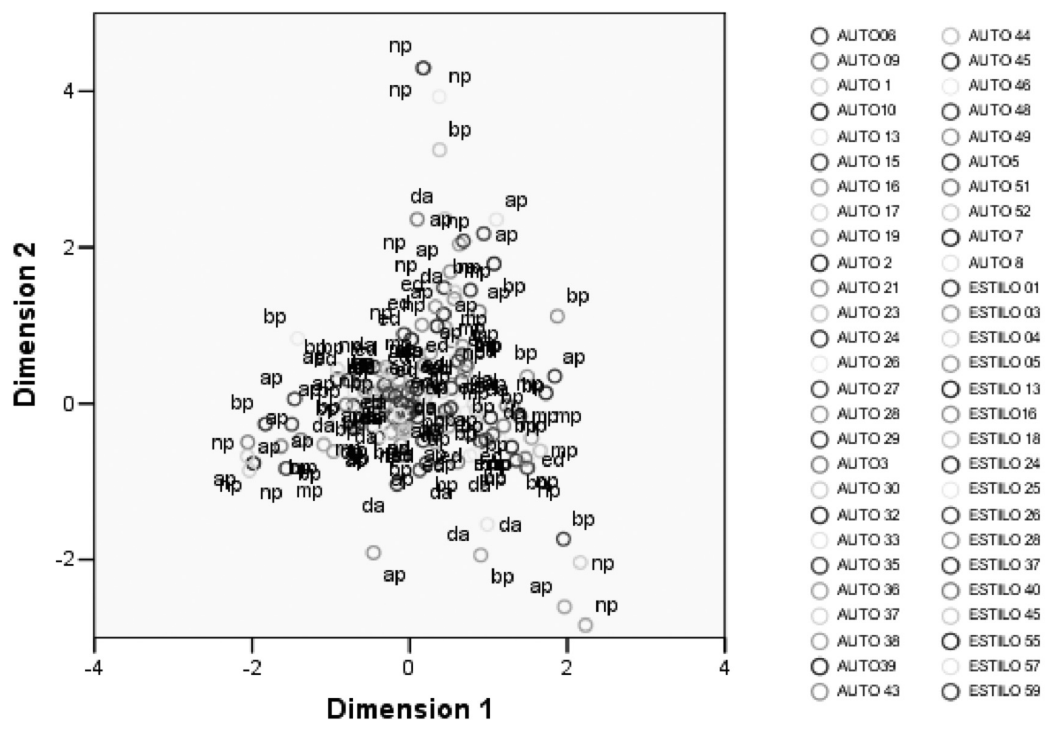

Figura N. ${ }^{\circ}$ 7. Mapa de correspondencias del área Económico-Empresariales.
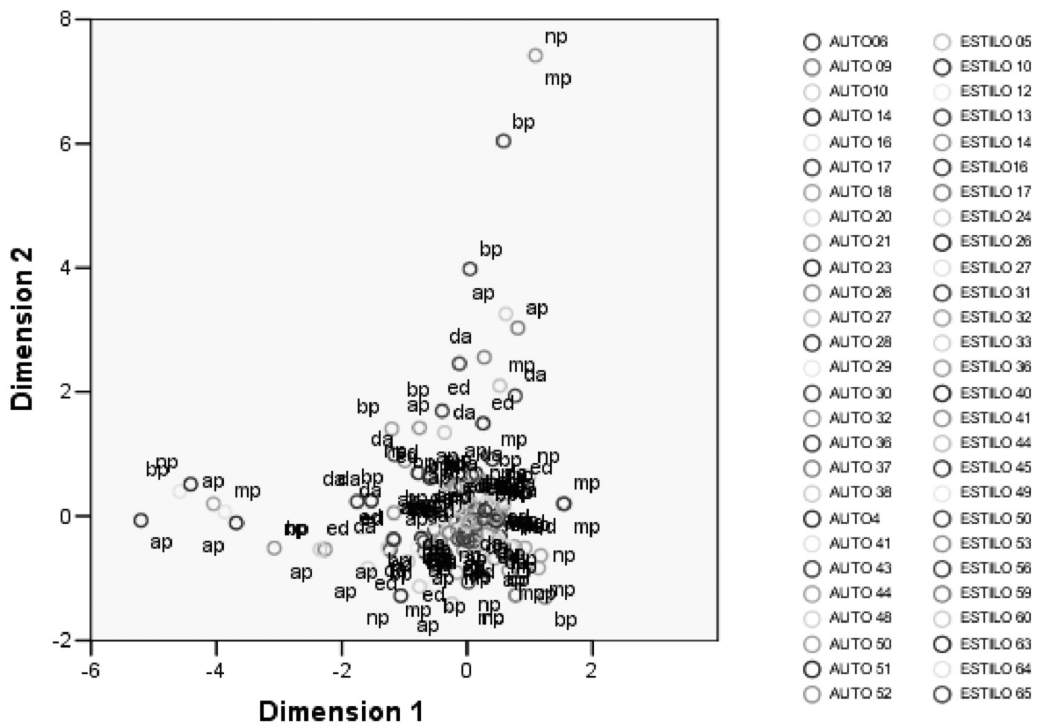

Figura N. ${ }^{\circ}$. Mapa de correspondencias del área de Humanidades. 


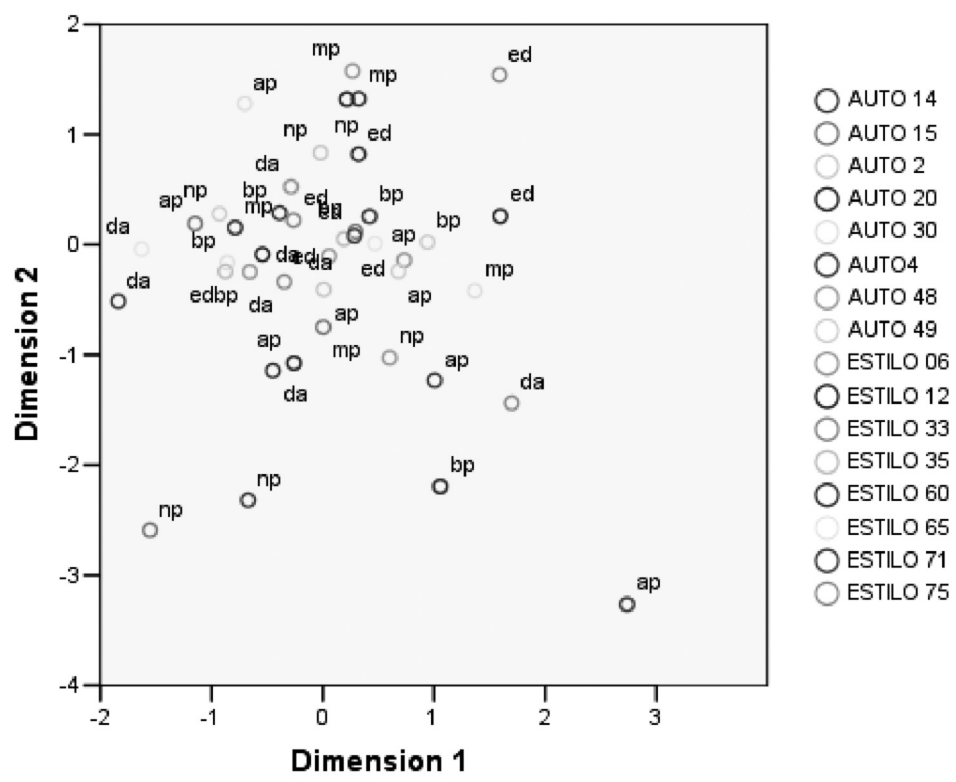

Figura N. ${ }^{\circ}$ 9. Mapa de correspondencias del área de Ingeniería.

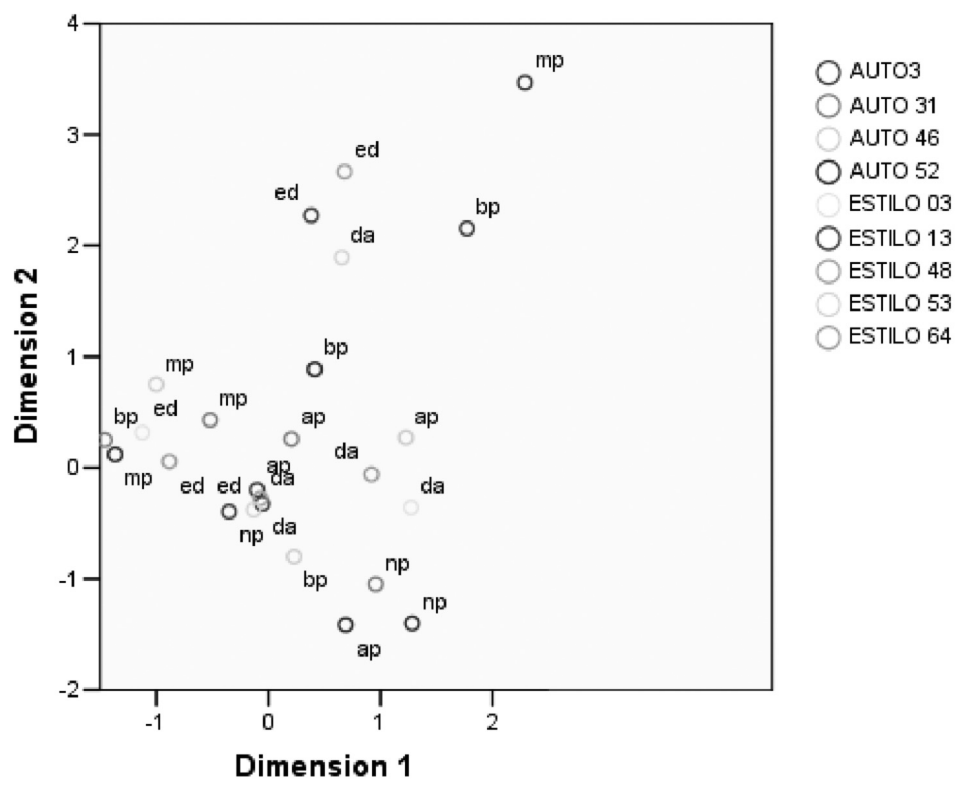

Figura N. ${ }^{0}$ 10. Mapa de correspondencias del área ciencias de la Salud. 
Tabla N. ${ }^{0}$ 6. Consolidado del mapa de correspondencias.

\begin{tabular}{|c|c|c|c|c|}
\hline Ciencias Basicas & Empresariales & Humanidades & Ingenierias & Ciencias Salud \\
\hline AUTO 4 & AUTO 1 & AUTO 4 & AUTO 2 & AUTO 3 \\
\hline AUTO 5 & AUTO 2 & AUTO 06 & AUTO 4 & AUTO 31 \\
\hline AUTO 6 & AUTO 3 & AUTO 09 & AUTO 14 & AUTO 46 \\
\hline AUTO 7 & AUTO 5 & AUT010 & AUTO 15 & AUTO 52 \\
\hline AUTO 8 & AUTO 06 & AUTO 14 & AUTO 20 & ESTILO 03 \\
\hline AUT010 & AUTO 7 & AUTO 16 & AUTO 30 & ESTILO 13 \\
\hline AUTO 20 & AUTO 8 & AUTO 17 & AUTO 48 & ESTILO 48 \\
\hline AUTO 21 & AUTO 09 & AUTO 18 & AUTO 49 & ESTILO 53 \\
\hline AUTO 22 & AUT010 & AUTO 20 & ESTILO 06 & ESTILO 64 \\
\hline AUTO 24 & AUTO 13 & AUTO 21 & ESTILO 12 & \\
\hline AUTO 28 & AUTO 15 & AUTO 23 & ESTILO 33 & \\
\hline AUTO 29 & AUTO 16 & AUTO 26 & ESTILO 35 & \\
\hline AUTO 30 & AUTO 17 & AUTO 27 & ESTILO 60 & \\
\hline AUTO 32 & AUTO 19 & AUTO 28 & ESTILO 65 & \\
\hline AUTO 35 & AUTO 21 & AUTO 29 & ESTILO 71 & \\
\hline AUTO 37 & AUTO 23 & AUTO 30 & ESTILO 75 & \\
\hline AUTO 40 & AUTO 24 & AUTO 32 & & \\
\hline AUTO 42 & AUTO 26 & AUTO 36 & & \\
\hline AUTO 44 & AUTO 27 & AUTO 37 & & \\
\hline AUTO 46 & AUTO 28 & AUTO 38 & & \\
\hline AUTO 47 & AUTO 29 & AUTO 41 & & \\
\hline AUTO 48 & AUTO 30 & AUTO 43 & & \\
\hline AUTO 49 & AUTO 32 & AUTO 44 & & \\
\hline AUTO 51 & AUTO 33 & AUTO 48 & & \\
\hline AUTO 52 & AUTO 35 & AUTO 50 & & \\
\hline ESTILO 8 & AUTO 36 & AUTO 51 & & \\
\hline ESTILO 10 & AUTO 37 & AUTO 52 & & \\
\hline ESTILO 12 & AUTO 38 & ESTILO 05 & & \\
\hline ESTILO 15 & AUTO39 & ESTILO 10 & & \\
\hline ESTILO 16 & AUTO 43 & ESTILO 12 & & \\
\hline ESTILO 18 & AUTO 44 & ESTILO 13 & & \\
\hline ESTILO 19 & AUTO 45 & ESTILO 14 & & \\
\hline ESTILO 21 & AUTO 46 & ESTILO 16 & & \\
\hline ESTILO 22 & AUTO 48 & ESTILO 17 & & \\
\hline ESTILO 23 & AUTO 49 & ESTILO 24 & & \\
\hline ESTILO 26 & AUTO 51 & ESTILO 26 & & \\
\hline ESTILO 27 & AUTO 52 & ESTILO 27 & & \\
\hline ESTILO 31 & ESTILO 01 & ESTILO 31 & & \\
\hline ESTILO 32 & ESTILO 03 & ESTILO 32 & & \\
\hline ESTILO 34 & ESTILO 04 & ESTILO 33 & & \\
\hline ESTILO 35 & ESTILO 05 & ESTILO 36 & & \\
\hline ESTILO 36 & ESTILO 13 & ESTILO 40 & & \\
\hline ESTILO 37 & ESTILO 16 & ESTILO 41 & & \\
\hline ESTILO 38 & ESTILO 18 & ESTILO 44 & & \\
\hline ESTILO 40 & ESTILO 24 & ESTILO 45 & & \\
\hline ESTILO 43 & ESTILO 25 & ESTILO 49 & & \\
\hline ESTILO 49 & ESTILO 26 & ESTILO 50 & & \\
\hline ESTILO 50 & ESTILO 28 & ESTILO 53 & & \\
\hline ESTILO 53 & ESTILO 37 & ESTILO 56 & & \\
\hline ESTILO 55 & ESTILO 40 & ESTILO 59 & & \\
\hline ESTILO 59 & ESTILO 45 & ESTILO 60 & & \\
\hline ESTILO 61 & ESTILO 55 & ESTILO 63 & & \\
\hline ESTILO 62 & ESTILO 57 & ESTILO 64 & & \\
\hline ESTILO 69 & ESTILO 59 & ESTILO 65 & & \\
\hline ESTILO 70 & ESTILO 60 & ESTILO 77 & & \\
\hline ESTILO 71 & ESTILO 62 & ESTILO 78 & & \\
\hline ESTILO 72 & ESTILO 63 & ESTILO 79 & & \\
\hline ESTILO 73 & ESTILO 66 & ESTILO 80 & & \\
\hline ESTILO 74 & ESTILO 70 & & & \\
\hline ESTILO 75 & ESTILO 71 & & & \\
\hline ESTILO 76 & ESTILO 74 & & & \\
\hline \multirow[t]{2}{*}{ ESTILO 78} & ESTILO 77 & & & \\
\hline & ESTILO 79 & & & \\
\hline
\end{tabular}




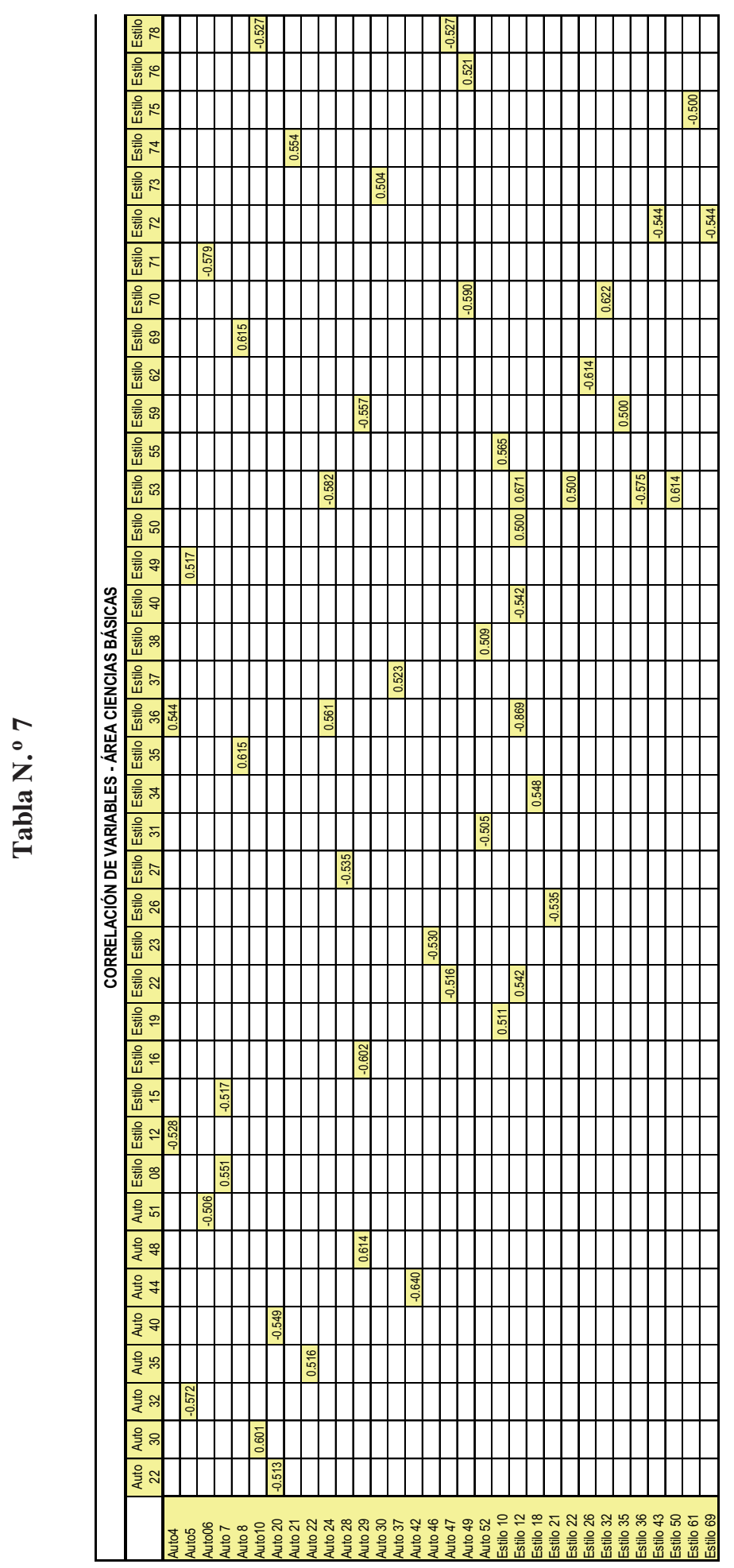




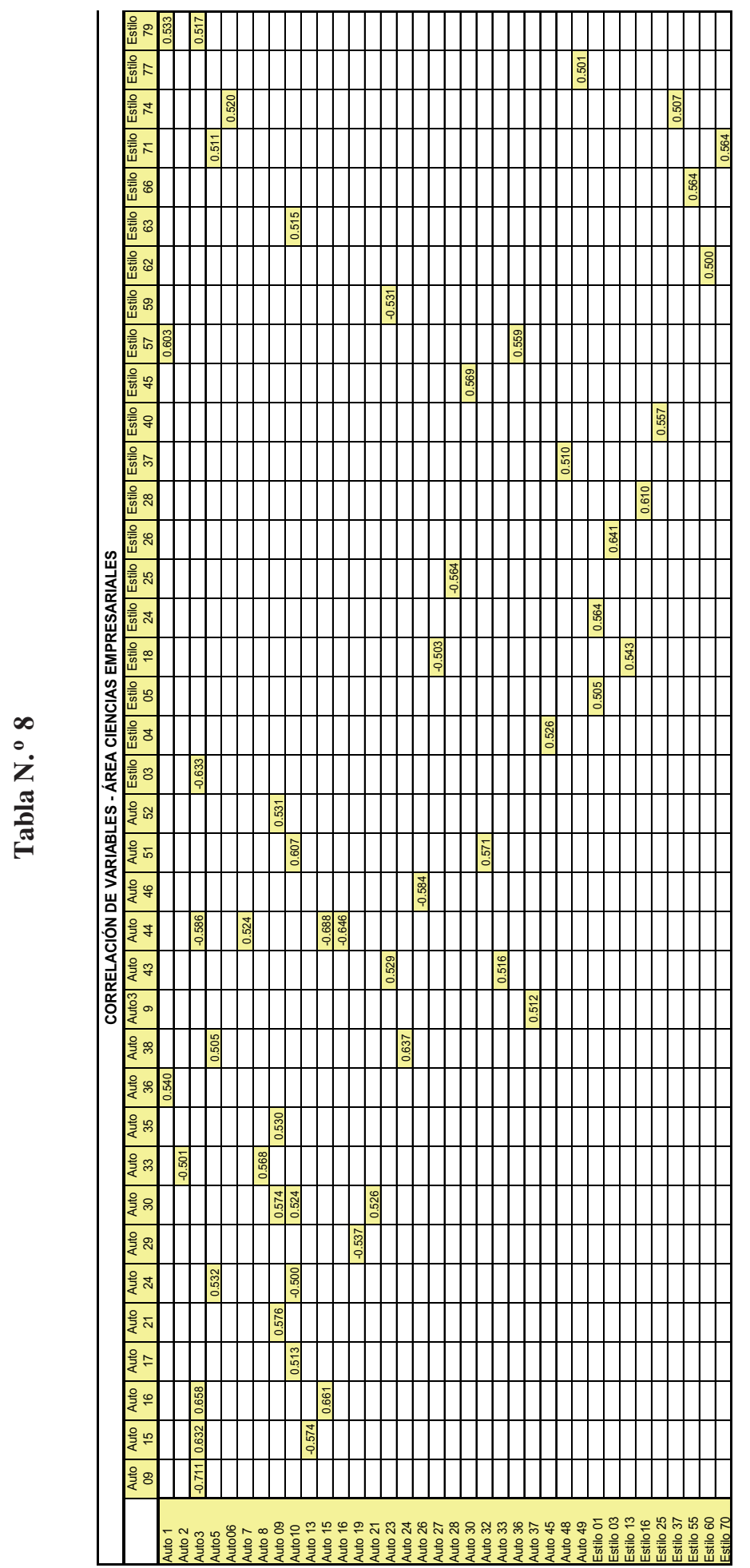


Oswaldo Orellana M. et al.

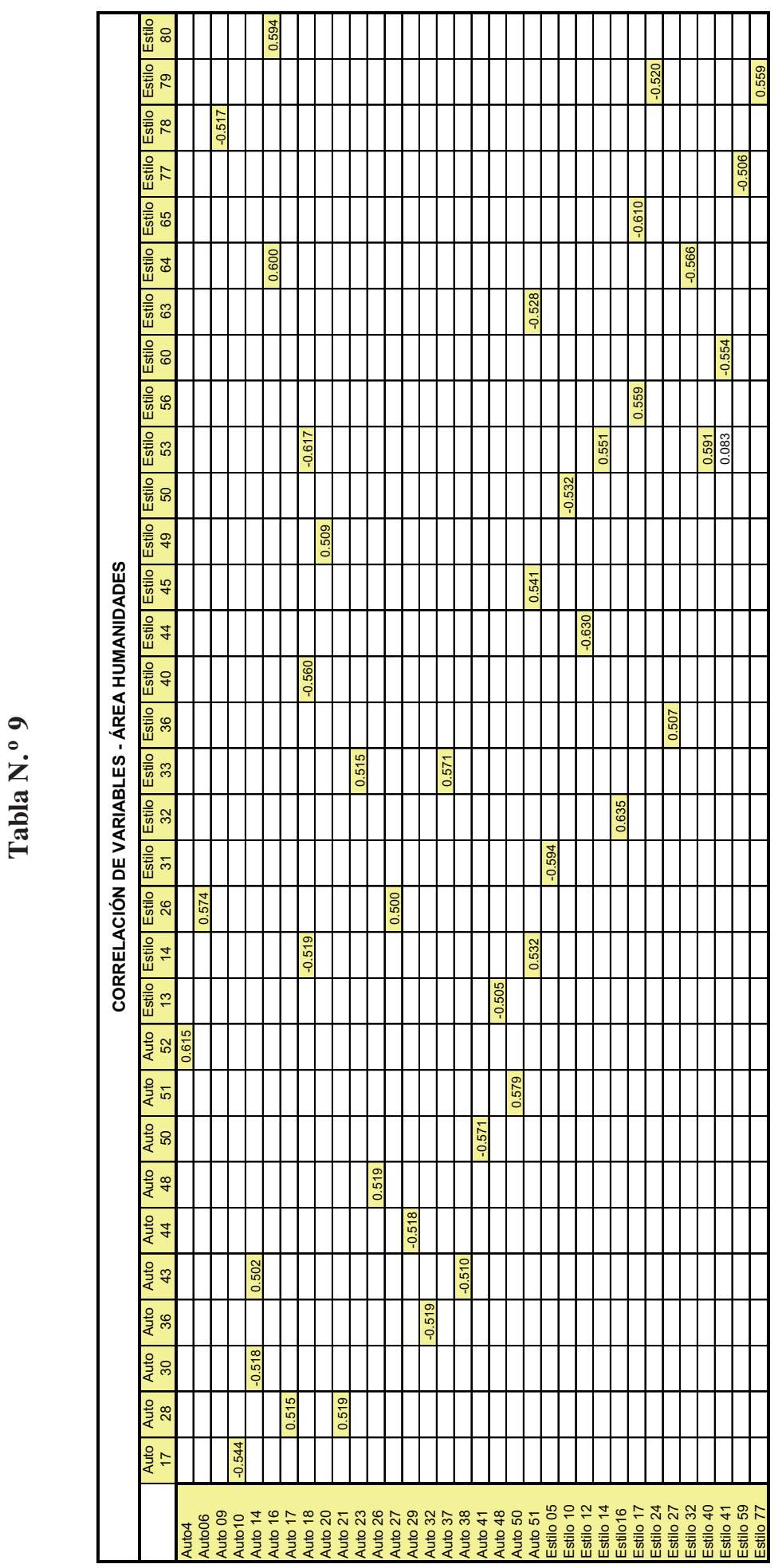

Revista de InVestigación en Psicología - Vol. 12, N. ${ }^{0} 2$ 
Tabla N. ${ }^{\circ} 10$

\begin{tabular}{|c|c|c|c|c|c|c|c|c|c|}
\hline \multicolumn{10}{|c|}{ CORRELACIÓN DE VARIABLES - ÁREA INGENIERÍAS } \\
\hline & Auto 20 & Auto 49 & Estilo 06 & Estilo 12 & Estilo 33 & Estilo 60 & Estilo 65 & Estilo 71 & Estilo 75 \\
\hline \multirow{9}{*}{$\begin{array}{l}\text { Auto } 2 \\
\text { Auto } 4 \\
\text { Auto } 14 \\
\text { Auto } 15 \\
\text { Auto } 20 \\
\text { Auto } 30 \\
\text { Auto } 48 \\
\text { Estilo } 12 \\
\text { Estilo } 35\end{array}$} & & & 0.548 & & & & & & \\
\hline & & & & & 0.556 & & & & \\
\hline & -0.541 & & & & & & & & \\
\hline & & & & & & & 0.548 & & \\
\hline & & & & & & -0.609 & & & \\
\hline & & & & 0.560 & & & & & \\
\hline & & -0.516 & & & & & & & \\
\hline & & & & & & & & & 0.533 \\
\hline & & & & & & & & -0.615 & \\
\hline
\end{tabular}

Tabla N. ${ }^{0} 11$

\begin{tabular}{|l|c|c|c|c|c|}
\hline \multicolumn{7}{|c|}{ CORRELACIÓN DE VARIABLES - ÁREA CIENCIAS DE LA SALUD } \\
\hline & Auto 52 & Estilo 03 & Estilo 48 & Estilo 53 & Estilo 64 \\
\hline Auto3 & & & & -0.524 & \\
Auto 31 & 0.523 & 0.512 & & & \\
Auto 46 & & 0.545 & & & \\
Auto 52 & & 0.620 & & & \\
Estilo 03 & & & & & 0.542 \\
Estilo 13 & & & 0.527 & & \\
\cline { 2 - 6 } & & & & & \\
\hline
\end{tabular}

